Review

\title{
Q1 Parkinson's disease: Autoimmunity and neuroinflammation
}

\section{De Virgilio Armando ${ }^{\mathrm{a}, \mathrm{b}}$, Greco Antonio a , Fabbrini Giovanni ${ }^{\mathrm{c}}$, Inghilleri Maurizio ${ }^{\mathrm{c}}$, Rizzo Maria Ida ${ }^{\mathrm{a}, \mathrm{b}, *}$,} Gallo Andrea ${ }^{\mathrm{d}}$, Conte Michela ${ }^{\mathrm{a}}$, Rosato Chiara ${ }^{\mathrm{d}}$, Ciniglio Appiani Mario ${ }^{\mathrm{a}, \mathrm{b}}$, de Vincentiis Marco ${ }^{\mathrm{a}}$

a Department Organs of Sense, ENT Section, 'Sapienza' University of Rome, Viale del Policlinico 155, 00100, Rome, Italy

b Department of Surgical Science, 'Sapienza' University of Rome, Viale del Policlinico 155, 00100, Rome, Italy

c Department of Neurology and Psychiatry, 'Sapienza' University of Rome, Viale del Policlinico 155, 00100, Rome, Italy

d Department of Medico-Surgical Sciences and Biotechnologies, Otorhinolaryngology Section, 'Sapienza' University of Rome, Corso della Repubblica, 79,04100 Latina, Italy

A R T I C L E I N F O

Available online $\mathrm{xxxx}$

\section{Keywords:}

Parkinson

Neurodegenerative disease

Autoimmunity

Inflammation

$\alpha$-Synuclein

Lewy bodies

Olfactory dysfunction

\begin{abstract}
A B S T R A C T
Parkinson's disease is a neurodegenerative disease that causes the death of dopaminergic neurons in the 18 substantia nigra. The resulting dopamine deficiency in the basal ganglia leads to a movement disorder that is 19 characterized by classical parkinsonian motor symptoms. Parkinson's disease is recognized as the most common 20 neurodegenerative disorder after Alzheimer's disease.

PD ethiopathogenesis remains to be elucidated and has been connected to genetic, environmental and immuno- 22 logic conditions.

The past decade has provided evidence for a significant role of the immune system in PD pathogenesis, either 24 through inflammation or an autoimmune response. Several autoantibodies directed at antigens associated 25 with PD pathogenesis have been identified in PD patients. This immune activation may be the cause of, rather 26 than a response to, the observed neuronal loss.

Parkinsonian motor symptoms include bradykinesia, muscular rigidity and resting tremor. The non-motor fea- 28 tures include olfactory dysfunction, cognitive impairment, psychiatric symptoms and autonomic dysfunction. 29 Microscopically, the specific degeneration of dopaminergic neurons in the substantia nigra and the presence of 30 Lewy bodies, which are brain deposits containing a substantial amount of $\alpha$-synuclein, have been recognized. 31 The progression of Parkinson's disease is characterized by a worsening of motor features; however, as the disease 32 progresses, there is an emergence of complications related to long-term symptomatic treatment.

The available therapies for Parkinson's disease only treat the symptoms of the disease. A major goal of Parkinson's 34 disease research is the development of disease-modifying drugs that slow or stop the neurodegenerative process. 35 Drugs that enhance the intracerebral dopamine concentrations or stimulate dopamine receptors remain the 36 mainstay treatment for motor symptoms.

Immunomodulatory therapeutic strategies aiming to attenuate PD neurodegeneration have become an attractive 38 option and warrant further investigation.
\end{abstract}

\section{Contents}

1. Introduction

2. Epidemiology . . . . . . . . . . . . . . . . . . . . . . . . . . . . . . . . . . . . 0

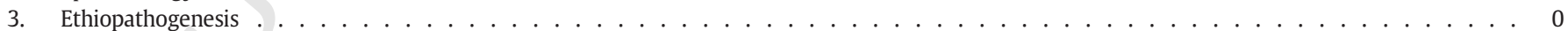

4. Clinical features . . . . . . . . . . . . . . . . . . . . . . . . . . . . . . . . . . . . . . . . . . . . 0

5. Histopathology. . . . . . . . . . . . . . . . . . . . . . . . . . . . . . . . . . . . . 0

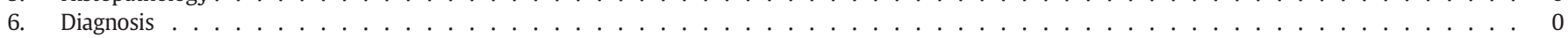

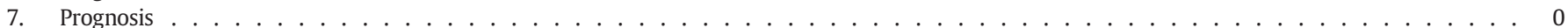

8. Treatment. . . . . . . . . . . . . . . . . . . . . . . . . . . . . . . . . . . . . . . . 0

\footnotetext{
* Corresponding author at: Department of Surgical Sciences, University of Rome 'La Sapienza', Viale del Policlinico 155, 00100 Rome, Italy. Fax: 0649976803.

E-mail address: mariaidarizzo@gmail.com (R.M. Ida).
} 


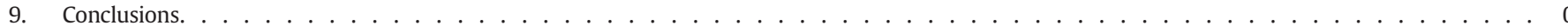

Take-home messages . . . . . . . . . . . . . . . . . . . . . . . . . . . . . . . . . . . . . . . . 0

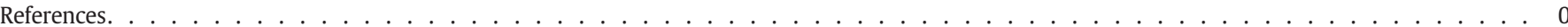

\section{Introduction}

Parkinson's disease is a neurodegenerative disease that results in the death of dopaminergic neurons in the substantia nigra pars compacta (SNpc). The resulting dopamine deficiency within the basal ganglia leads to a movement disorder characterized by classical parkinsonian motor symptoms.

Parkinson's disease was first medically described as a neurological syndrome by James Parkinson in 1817, although some aspects of Parkinson's disease were reported in earlier descriptions [1]. For example, Sylvius de la Boë wrote of resting tremor and Sauvages described festination [2,3]. Much earlier, traditional Indian texts from approximately $1000 \mathrm{BC}$ and ancient Chinese sources also provided descriptions that were reminiscent of Parkinson's disease [4,5]. Over 50 years later, Jean-Martin Charcot was more thorough in his descriptions and distinguished bradykinesia as a separate cardinal feature of the illness [6].

\section{Epidemiology}

Parkinson's disease is recognized as the most common neurodegenerative disorder after Alzheimer's disease [7,8]. The incidence of Parkinson's disease ranges from 10 to 18 per 100,000 person-years [9]. Gender is an established risk factor, with a male-to-female ratio of approximately 3:2 [10]. Ethnicity is also a risk factor for the disease. In the USA, the incidence is highest in people of Hispanic ethnic origin, followed by non-Hispanic Whites, Asians and Blacks [9]. Age is the greatest risk factor for the development of Parkinson's disease. The prevalence and incidence increase nearly exponentially with age and peak after 80 years of age $[11,12]$. This trend has important public health implications; as the aging population and life expectancy increase worldwide, the number of people with Parkinson's disease is expected to increase by more than $50 \%$ by 2030 [7].

\section{Ethiopathogenesis}

Currently, PD ethiopathogenesis remains to be elucidated, and the destruction of dopaminergic neurons in PD has been connected to a variety of factors, including genetic, environmental and immunological 99 conditions.

Genetic factors have been identified in familiar forms of PD, which 101 contribute to approximately 10\% of PD cases [13,14]. Environmental fac- 102 tors that were shown to be associated with a decreased risk were tobac- 103 co smoking, coffee drinking, non-steroidal anti-inflammatory drug use, 104 calcium channel blocker use, and alcohol consumption [15]. Factors that 105 increase the risk of developing PD were pesticide exposure, prior head 106 injury, rural living, $\beta$-blocker use, agricultural occupation, and well- 107 water drinking [15].

Furthermore, the results of epidemiological studies [15] showed that 109 the use of anti-inflammatory medications, specifically non-steroidal 110 anti-inflammatory drugs, reduced the risk of developing Parkinson's 111 disease, supporting the hypothesis that inflammation might promote 112 an underlying disease process (Fig. 1).

113

Currently, PD etiopathogenesis remains to be elucidated. Recently, 114 reviews of the current literature have brought to light evidence for the 115 possible role of the immune system, specifically autoimmune mecha- 116 nisms, in the ethiopathogenesis of PD [16]. Previously, it was believed 117 that PD is not mediated by autoimmune mechanisms [17]. However, 118 data accumulated over the past decade regarding immune alterations 119 in PD increased the interest in pursuing such an association. A series 120 of independent observations has led to the convergence of the view 121 that innate and adaptive immune mechanisms might play a role in the 122 development of PD [18].

Neuroinflammation is a characteristic feature of Parkinson's 124 disease pathology, but it has yet to be established whether neuro- 125 inflammation promotes or protects from neurodegeneration. A 126 significant increase in the level of innate immune components, in- 127 cluding complement and cytokines (e.g., IL-1, IL-2, IL-6, and TNF), 128 in the substantia nigra and cerebrospinal fluid (CSF) of PD patients 129 has been observed [18]. Elevation of $\gamma / \delta+$ T cells in the peripheral 130 blood and CSF of PD patients was also reported [19]. Benkler et al. 131 [20] then further pursued this quest and found evidence suggest- 132 ing that an autoimmune mechanism, which may be mediated via 133 humoral responses, might play a role in the ethiopathogenesis of 134 PD.

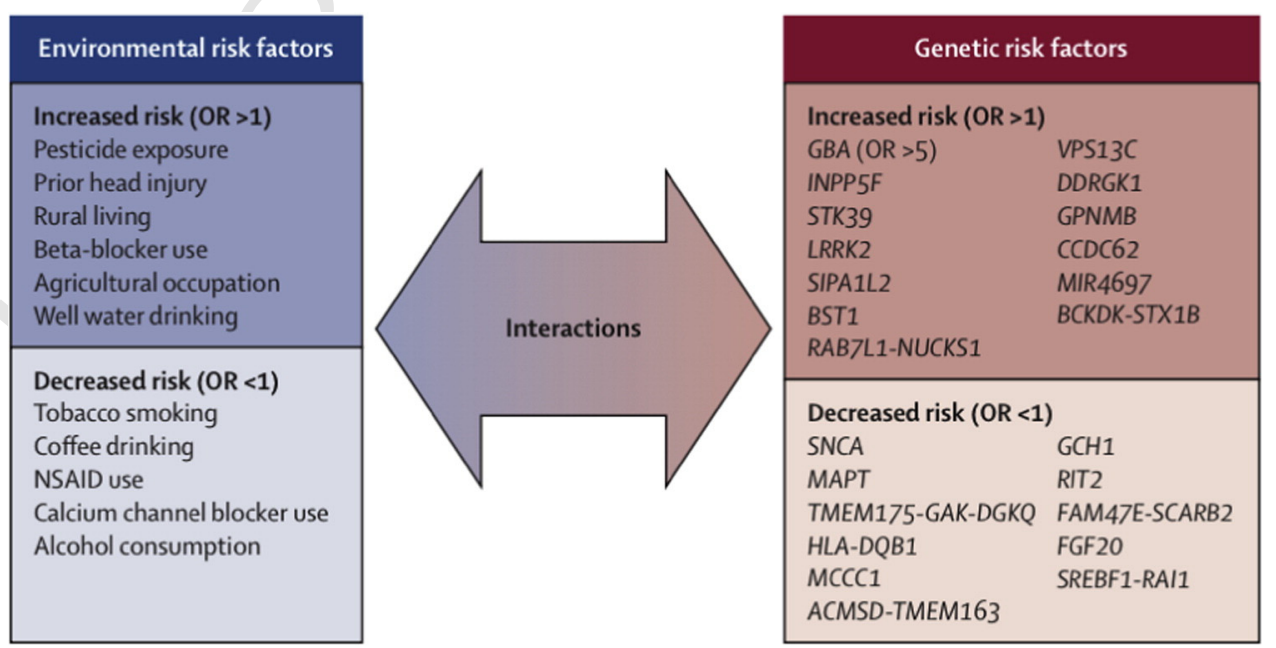

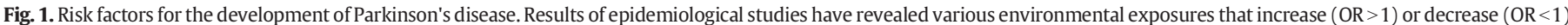

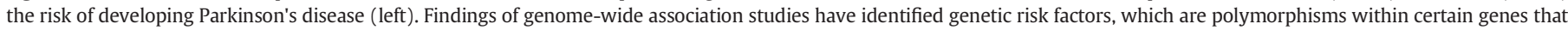

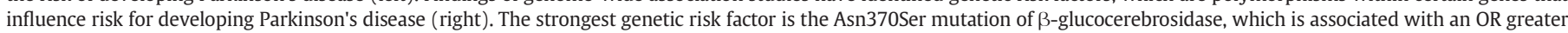
than 5. The interplay between environmental and genetic risk factors is under investigation. OR = odds ratio. (From: Lancet 2015;386:896:912). 
Over the last decade, several autoantibodies directed at antigens associated with or related to PD pathogenesis have been identified in PD patients, including antibodies directed at melanin [21], a-synuclein [22,23], and GM1 ganglioside [24].

Interestingly, a recent placebo-controlled study demonstrated that GM1-ganglioside supplementation was effective in improving tremorrelated motor functions, thus supporting a possible role for these antibodies in the pathogenesis of tremor in PD [24].

Autoreactive antibodies associated with PD have been found in the plasma and brain; a post-mortem analysis of brains from PD patients and controls showed that IgG was bound to dopaminergic neurons in tissues from patients with PD [25].

One potential target structure for an immune attack against dopaminergic neurons is the pigment neuromelanin (NM) that accumulates in dopaminergic neurons as a by-product of catecholamine metabolism [26].

Oberlander et al. recently showed that NM triggers the functional DC maturation in vitro, as NM-treated DCs were able to trigger a proliferative T cell response. They also showed that DCs can phagocytose NM [27].

These experiments demonstrate that the first criterion for DCs to initiate an adaptive autoimmune response directed against NM-associated structures was fulfilled. Koutsilieri et al. [28] hypothesize that activated DCs migrate from the brain into the cervical lymph node, where they present the potential (auto-)antigens to $\mathrm{T}$ and $\mathrm{B}$ cells. The recognition of NM as a pathogen or dangerous molecule and its uptake by DCs would allow the DCs to migrate, and its presentation in the cervical lymph nodes triggers an adaptive autoimmune response if NMreactive $\mathrm{T}$ or $\mathrm{B}$ cells are present. This autoimmune response against NM would be directed against NM-rich cells in the brain, leading to dopaminergic cell death. This auto-aggressive loop would be enhanced by NM-triggered activation of microglia [29,30], resulting in an amplification of the adaptive immune response against NM and the local reactivation of the immigrating effector T cells (Fig. 2). There is accumulating evidence for an immunogenic role of NM in PD pathogenesis. Antibodies directed at catecholamine-based melanins have been detected in the sera from PD patients [21].
In another study, CSF derived auto-Abs that react with dopaminergic 172 neurons in the substantia nigra were present in 78\% of patients com- 173 pared with $3 \%$ of the controls [31].

Moreover, the CSF of PD patients exerted a cytotoxic effect on dopa- 175 minergic neurons, which enhanced the substantia nigra degeneration in 176 a time- and dose-dependent manner [31,32]. This cytotoxic effect was 177 further demonstrated by neuronal labelling with IgG, which correlated 178 with neurodegeneration in PD [25].

Early experimental evidence in favour of an autoimmune back- 180 ground of PD came from Chen et al., who reported that the transfer of 181 plasma antibodies isolated from PD patients to the substantia nigra of 182 rats induced a marked loss of dopaminergic neurons. In contrast, ani- 183 mals that were treated with antibodies from healthy controls exhibited 184 much less neuronal damage, suggesting that autoantibodies that recog- 185 nize dopaminergic cells are present in patients with PD [33]. 186

Furthermore, several immune-mediated mechanisms were pro- 187 posed to explain the possible mechanisms by which autoantibodies 188 may induce dopaminergic cell death, such as activation of apoptosis, 189 enhanced complement function, accelerated attack of the surround- 190 ing microglia cells [31,32,34,35], and competitive binding inhibition 191 [36,37].

Microglia cells, the tissue macrophage population of the brain, are 193 the main glial cell type that participates in the inflammatory response 194 in the brain. Microglial activation through an FC $\gamma$ receptor pathway 195 can be induced by IgG derived from PD patients, which subsequently 196 causes substantia nigra cell injury [34]. The plausible notorious effect 197 of antibodies on the dopaminergic system was further highlighted in a 198 series of in vitro and in vivo studies utilizing synthetic antibodies 199 (Abs) [36]. These engineered Abs were able to bind native dopamine re- 200 ceptors and competitively inhibit the dopamine receptors' ability to 201 bind their natural agonists [37,38].

Taken together, these results might suggest a role for autoanti- 203 bodies, which are a prominent feature of autoimmunity, in the 204 ethiopathogenesis of PD. However, recent studies suggest that this 205 immune activation may be the cause of, rather than a response to, 206 the observed neuronal loss.

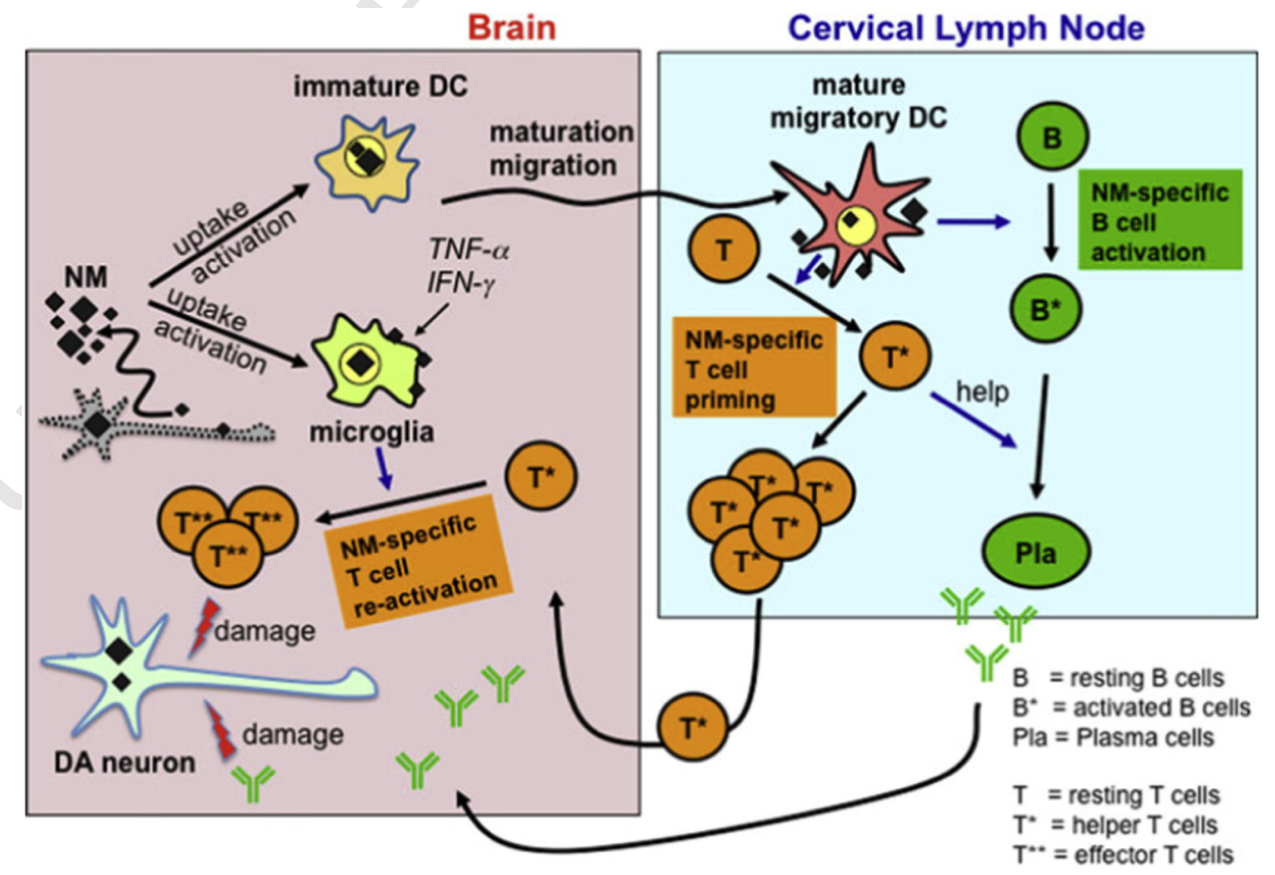

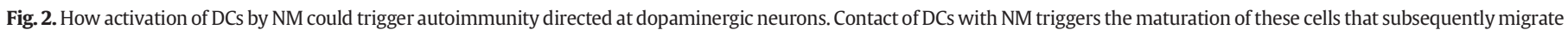
from the brain into the cervical lymph nodes where they present NM to B- and T-lymphocytes. 


\section{Clinical features}

Parkinsonian motor symptoms include bradykinesia, muscular rigidity and resting tremor [39]. The non-motor features include olfactory dysfunction, cognitive impairment, psychiatric symptoms, sleep disorders and autonomic dysfunction.

Non-motor features are also frequently present in Parkinson's disease before the onset of the classical motor symptoms (Fig. 1) [40]. The premotor phase can be prolonged; for example, the average latency between the onset of the early symptoms and occurrence of parkinsonian motor symptoms is $12-14$ years [40].

In late-stage Parkinson's disease, treatment-resistant motor and non-motor features are prominent and include axial motor symptoms, such as postural instability, freezing of gait, falls, dysphagia, and speech dysfunction. After approximately 17 years of disease, up to $80 \%$ of patients with Parkinson's disease have a freezing of gait and falls, and up to $50 \%$ of patients report choking [41]. Dementia is particularly prevalent, occurring in $83 \%$ of patients with Parkinson's disease who have had a disease duration of 20 years [42] (Fig. 3).

\section{Histopathology}

The loss of dark pigmentation in the substantia nigra and frontal atrophy are typical examples of macroscopic brain aberrations that develop in PD [43].

Microscopically, two predominant features have been recognized: a specific degeneration of dopaminergic neurons in the substantia nigra and the presence of Lewy bodies, which are brain deposits that contain a substantial amount of $\alpha$-synuclein [43]. In its misfolded state, $\alpha$-synuclein becomes insoluble and aggregates to form intracellular inclusions within the cell body (Lewy bodies) and processes (Lewy neurites) of neurons [44]. Lewy pathology is not restricted to the brain, but can also be found in the spinal cord and peripheral nervous system [45].

\section{Diagnosis}

A clinical diagnosis of Parkinson's disease is based on the presence of parkinsonian motor features, namely, bradykinesia, rigidity and resting tremor.

Strategies to develop biomarkers for the diagnosis of Parkinson's disease are under investigation, particularly to enable diagnosis early in the disease course, even before the onset of motor symptoms. Potential clinical markers include olfactory impairment, as measured by standard methods, such as the University of Pennsylvania's smell identification 247 test [40]. The proposed pathological markers are being tested on the 248 basis of earlier findings of $\alpha$-synuclein within the peripheral nervous 249 system. The concentrations of $\alpha$-synuclein, DJ-1, tau and $\beta$-amyloid 250 $[46,47]$, as well as the $\beta$-glucocerebrosidase activity in the cerebrospinal 251 fluid are being tested as potential biochemical biomarkers of early 252 Parkinson's disease [48,49] (Fig. 4). 253

Candidate imaging markers include positron emission tomography 254 (PET) or single photon emission computed tomography (SPECT) 255 methods to measure the reduction in the number of SNpc dopaminergic 256 nerve terminals projecting to the striatum [50]. Standard MRI has a mar- 257 ginal role in Parkinson's disease diagnosis, but high and ultra-high-field 258 (7 Tesla) MRI combined with advanced techniques, such as diffusion 259 tensor imaging, are being explored for early diagnosis of Parkinson's 260 disease [51,52].

For people with family members with a known monogenic form of 262 Parkinson's disease, genetic testing can assist in the diagnosis. 263

A single measure might not suffice for an accurate and early diagno- 264 sis of such a complex disease. Instead, a combination of imaging, bio- 265 chemical and genetic biomarkers might be required.

\section{Prognosis}

The progression of Parkinson's disease is characterized by the 268 worsening of motor features, which can initially be managed with 269 symptomatic therapies. However, as the disease progresses, there is 270 an emergence of complications related to long-term symptomatic treat- 271 ment, including motor and non-motor fluctuations, dyskinesia and psy- 272 chosis [41]. Symptoms of late-stage Parkinson's disease substantially 273 contribute to disability and are strong predictors of a need for admission 274 to an institution and mortality [53].

\section{Treatment}

276

The available therapies for Parkinson's disease only treat the symp- 277 toms of the disease. A major goal of Parkinson's disease research is the 278 development of disease-modifying drugs that slow or stop the underly- 279 ing neurodegenerative process. Drugs that can slow or stop the neuro- 280 degenerative process in Parkinson's disease are not yet available, but 281 such disease-modifying drugs are anticipated to be most effective if pa- 282 tients can be diagnosed and treated during this prodromal premotor pe- 283 riod. Drugs that enhance intracerebral dopamine concentrations or 284 stimulate dopamine receptors remain the mainstay treatment for 285 motor symptoms. These drugs include levodopa, dopamine agonists, 286

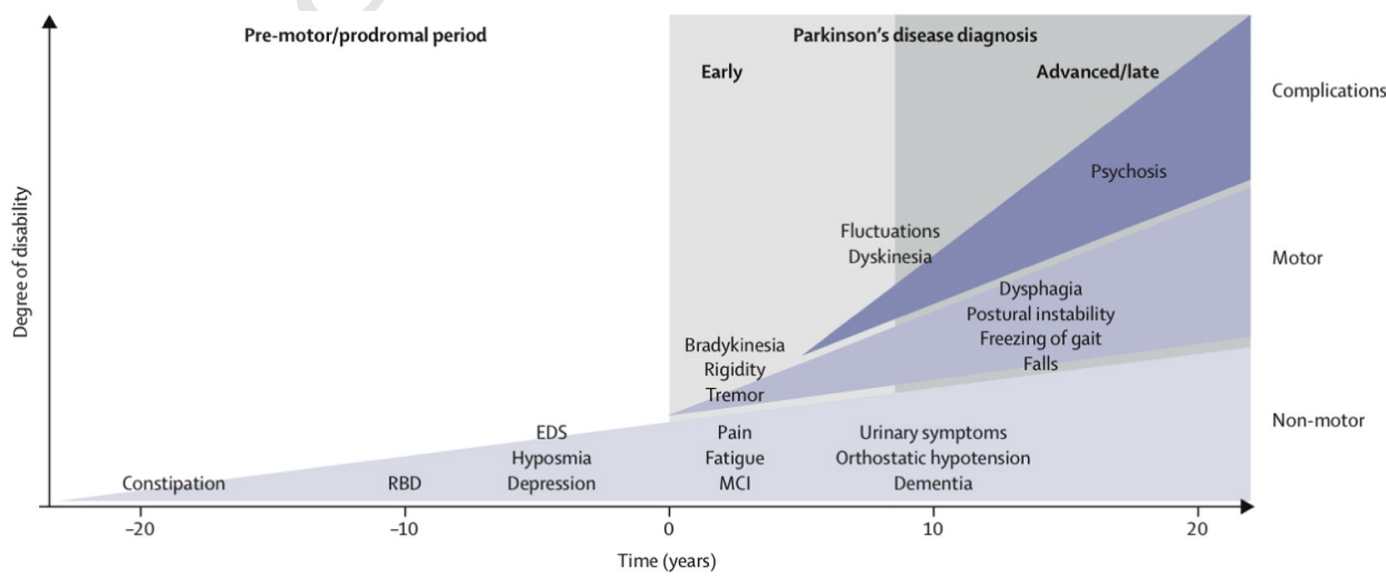

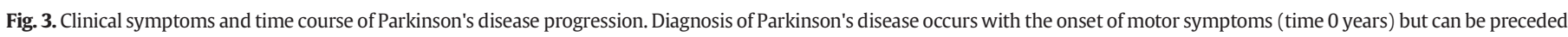

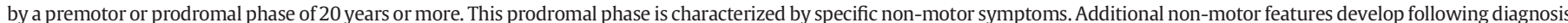

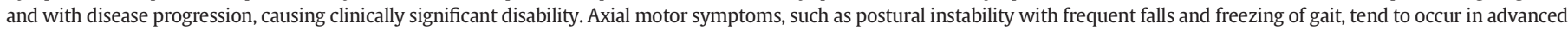

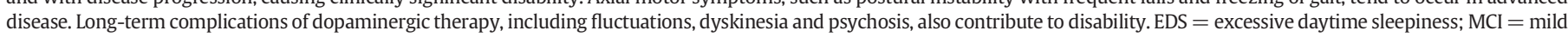
cognitive impairment; RBD = REM sleep behaviour disorder. (From: Lancet 2015;386:896:912). 


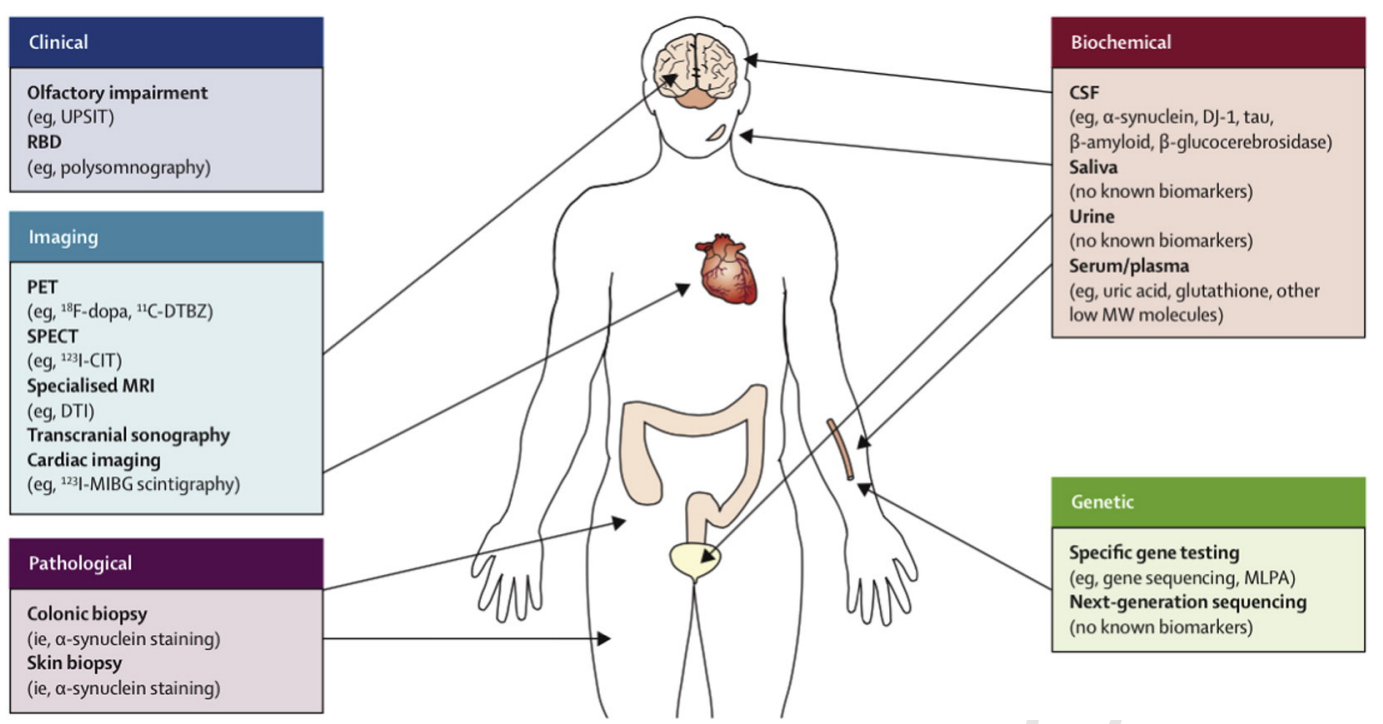

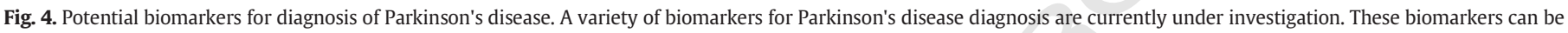

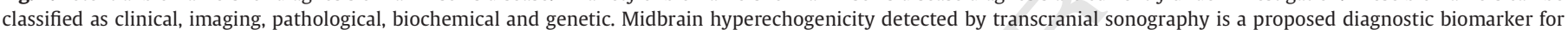

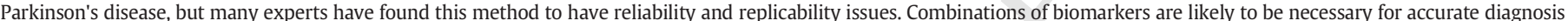

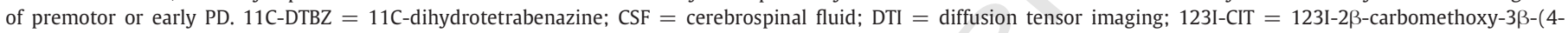

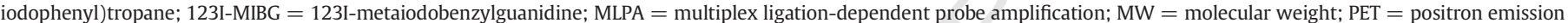

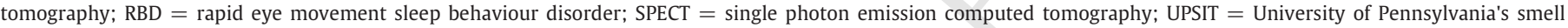
identification test. (From: Lancet 2015;386:896:912).

monoamine oxidase type B inhibitors and, less commonly, amantadine $[54,55]$. Because none of these drugs have proven to be neuroprotective or disease-modifying, therapy does not need to be started at the time of diagnosis for all patients. However, there is little justification for delay. Treatment should be initiated when symptoms cause disability or discomfort to the patient, with the goal of improving function and quality of life.

The past decade has provided accumulating evidence for a significant role of the immune system in PD pathogenesis, either through inflammation or an autoimmune response. Thus, immunomodulatory therapy strategies aiming to attenuate PD disease progression have become an attractive option and warrant further investigation. However, the negative results of non-steroidal anti-inflammatory drugs in late PD [56] strongly suggest that early immunomodulation is the key to preventing PD onset and progression.

Minocycline, a broad-spectrum tetracycline antibiotic, has been tested in experimental models and PD patients. Minocycline effectively crosses the blood-brain barrier (BBB) and shows potent anti-inflammatory effects in neurotoxin models of PD [57]. A randomized, double-blind, Phase II clinical trial showed that minocycline offers a clinical benefit to early PD patients, which warrants further consideration of minocycline for use in Phase III clinical trials [58].

Leucine-rich repeat kinase 2 (LRRK2) is an enzyme that is highly expressed in peripheral macrophages and monocytic cells, as well as central microglia, suggesting a functional role for LRRK2 in the innate immune system $[59,60]$. Inhibition or attenuation of LRRK2 is a promising therapeutic strategy as an anti-inflammatory treatment for PD.

Peroxisome proliferator-activated receptor gamma coactivator 1alpha (PGC- $1 \alpha$ ) is a potential new target for anti-inflammatory therapy in PD patients. PGC- $1 \alpha$ activity is mainly controlled by the peroxisome proliferator-activated receptors (PPARs), 5' AMP-activated protein kinase (AMPK), and sirtuin 1 (Sirt1) [61]. Hence, pharmacological activators for these proteins have the potential to exert anti-inflammatory effects by activating PGC- $1 \alpha$. These activators include fibrates and rosiglitazone (PPAR) [62,63], metformin [64], pyrroloquinoline quinone [65], 5-aminoimidazole-4-carboxamide ribonucleotide (AICAR) (AMPK) [66] and resveratrol (Sirt1) [67].
As PPAR agonists (fibrates and rosiglitazone) and AMPK activators 325 (metformin and AICAR) are already routinely used in clinical practice 326 for the treatment of metabolic syndrome and type 2 diabetes, these 327 drugs could be readily translated from animal models to PD patients. 328 Preclinical CNS distribution and efficacy studies using inflammatory an- 329 imal models of PD will be sufficient to warrant clinical trials on these 330 drugs.

\section{Conclusions}

Parkinson's disease is a debilitating disease of unknown cause, 333 despite major scientific and therapeutic advances. The extensive 334 damage to the dopaminergic system in PD seems to be intercon- 335 nected with genetics, environmental and immunological factors, 336 which is described as a mosaic of many autoimmune diseases 337 [68]. In this review, we pursued the evidence for immune- and 338 autoimmune-mediated mechanisms that are associated with PD. 339 A unique observation indicated that olfactory dysfunction may be 340 a consequence of an autoimmune mechanism. As decreased olfac- 341 tion is one of the earliest non-motor signs of PD, this observation 342 might shed more light on a possible association between autoim- 343 munity and PD.

The prevalence of several brain-associated autoantibodies in the sera 345 of PD patients further support the possible role of immunoglobulin- 346 mediated autoimmune mechanisms in the ethiopathogenesis of PD. 347 Not only can these autoantibodies serve as biomarkers of disease 348 but our renewed understanding of the nature of this complex dis- 349 ease might also be useful for the early diagnosis and treatment of 350 PD patients. It is even safe to say that novel therapeutics targeted 351 at specific autoantibodies may be able to differentiate between PD 352 subgroups. Further studies to evaluate a larger number of patients 353 and preferably a wider profile of brain-associated autoantibodies 354 might enable a better understanding of the precise ethiopathological 355 mechanism of PD.

Thus, although PD pathogenesis remains to be fully elucidated, it 357 seems that the inflammation and neuronal degeneration associated 358 with PD could be induced by autoimmune mechanisms, mainly via 359 brain-specific auto-Abs. 


\section{Take-home messages}

- Parkinson's disease is a neurodegenerative disease that causes the death of dopaminergic neurons in the substantia nigra pars compacta (SNpc). The resulting dopamine deficiency in the basal ganglia leads to a movement disorder that is characterized by classic parkinsonian motor symptoms.

- PD ethiopathogenesis remains to be elucidated and has been connected to genetic, environmental and immunological conditions. Recently, the role of autoimmune mechanisms in the ethiopathogenesis of PD has garnered more attention. Thus, it seems that neuronal degeneration could be induced by autoimmune mechanisms, mainly via brain-specific autoantibodies.

- Parkinsonian motor symptoms include bradykinesia, muscular rigidity and resting tremor. The non-motor features include olfactory dysfunction, cognitive impairment, psychiatric symptoms and autonomic dysfunction. Olfactory impairment is one of the first symptoms and allows an early diagnosis of PD many years before the onset of motor symptoms.

- Microscopically, two predominant features have been recognized: a specific degeneration of dopaminergic neurons in the substantia nigra and the presence of Lewy bodies, which are brain deposits containing a substantial amount of $\alpha$-synuclein.

- Therapies for Parkinson's disease only treat the symptoms of the disease. Drugs that enhance the intracerebral dopamine concentrations (levodopa) or stimulate dopamine receptors (dopamine agonists) remain the mainstay treatment for motor symptoms. A major goal of Parkinson's disease research is the development of disease-modifying drugs that stop the neurodegenerative process.

If NM-reactive lymphocytes are present, they get activated (primed) and secrete NM-specific antibodies (B cells) or exert NM-specific cytotoxic functions (T cells). Activation of microglia by NM would result in a proliferation of NM-specific T cells after contact with NMpresenting microglia. NM-specific antibodies and T cells may recognize NM-positive neurons and trigger their degradation. (From: J Neural Transm 2013;120:75-81).

\section{References}

[1] Parkinson J. An essay on the shaking palsy. Whittingham and Rowland for Sherwood. London: Needly and Jones; 1817.

[2] Sylvius de la Boë F. Opera Medica. Danielem Elsevirium et Abrahamum Wolfgang, Amsterdam; 1680.

[3] Tyler K. A history of Parkinson's disease. In: WC K, editor. Handbook of Parkinson's disease. New York: Marcel Dekker; 1992. p. 1-34.

[4] Manyam BV, Sánchez-Ramos JR. Traditional and complementary therapies in Parkinson's disease. Adv Neurol 1999;80:565-574.

[5] Zhang Z-X, Dong Z-H, GC R'n. Early descriptions of Parkinson's disease in ancient China. Arch Neurol 2006;63:782-784.

[6] Charcot J-M. On Parkinson's disease. Lectures on diseases of the nervous system delivered at the Salpêtrière. London: New Sydenham Society; 1877. p. 129-156.

[7] Dorsey ER, Constantinescu R, Thompson JP, Biglan KM, Holloway RG, Kieburtz K, et al. Projected number of people with Parkinson disease in the most populous nations, 2005 through 2030. Neurology 2007;68:384-386.

[8] Alzheimer's Association. 2014 Alzheimer's disease facts and figures. Alzheimers Dement 2014;10:e47-92.

[9] Van Den Eeden SK. Incidence of Parkinson's disease: variation by age, gender, and race/ethnicity. Am J Epidemiol 2003;157:1015-1022.

[10] De Lau LML, Breteler MMB. Epidemiology of Parkinson's disease. Lancet Neurol 2006;5:525-535.

[11] Driver JA, Logroscino G, Gaziano JM, Kurth T. Incidence and remaining lifetime risk of Parkinson disease in advanced age. Neurology 2009;72:432-438.

[12] Pringsheim T, Jette N, Frolkis A, Steeves TDL. The prevalence of Parkinson's disease: a systematic review and meta-analysis. Mov Disord 2014;29:1583-1590.

[13] Lesage S, Brice A. Parkinson's disease: from monogenic forms to genetic susceptibility factors. Hum Mol Genet 2009;18:R48-59.

[14] Rosner S, Giladi N, Orr-Urtreger A. Advances in the genetics of Parkinson's disease. Acta Pharmacol Sin 2008;29:21-34.

[15] Noyce AJ, Bestwick JP, Silveira-Moriyama L, Hawkes CH, Giovannoni G, Lees AJ, et al. Meta-analysis of early nonmotor features and risk factors for Parkinson disease. Ann Neurol 2012;72:893-901.
[16] Benkler M, Agmon-Levin N, Shoenfeld Y. Parkinson's disease, autoimmunity, and ol- 432 faction. Int J Neurosci 2009;119:2133-2143. 433

[17] Møller A, Perrild H, Pedersen H, Høier-Madsen M. Parkinson's disease and autoim- 434 munity. Acta Neurol Scand 1989;79:173-175. 435

[18] Liu B, Gao HM, Hong JS. Parkinson's disease and exposure to infectious agents and 436 pesticides and the occurrence of brain injury: role of neuroinflammation. Environ 437 Health Perspect 2003;111:1065-1073.

[19] Fiszer U, Mix E, Fredrikson S, Kostulas V, Olsson T, Link H. Gamma delta + T cells are 439 increased in patients with Parkinson's disease. J Neurol Sci 1994;121:39-45. 440

[20] Benkler M, Agmon-Levin N, Hassin-Baer S, Cohen OS, Ortega-Hernandez OD, Levy A, 441 et al. Immunology, autoimmunity, and autoantibodies in Parkinson's disease. Clin 442 Rev Allergy Immunol 2012;42:164-171.

[21] Double KL, Rowe DB, Carew-Jones FM, Hayes M, Chan DK, Blackie J, et al. Anti- 444 melanin antibodies are increased in sera in Parkinson's disease. Exp Neurol 2009; 445 217:297-301.

[22] Papachroni KK, Ninkina N, Papapanagiotou A, Hadjigeorgiou GM, Xiromerisiou G, 447 Papadimitriou A, et al. Autoantibodies to alpha-synuclein in inherited Parkinson's 448 disease. J Neurochem 2007;101:749-756. 449

[23] Yanamandra K, Gruden MA, Casaite V, Meskys R, Forsgren L, Morozova-Roche LA. 450 Alpha-synuclein reactive antibodies as diagnostic biomarkers in blood sera of 451 Parkinson's disease patients. PLoS One 2011;6:e18513.

24] Zappia M, Crescibene L, Bosco D, Arabia G, Nicoletti G, Bagala A, et al. Anti-GM1 gan- 453 glioside antibodies in Parkinson's disease. Acta Neurol Scand 2002;106:54-57. 454

[25] Orr CF, Rowe DB, Mizuno Y, Mori H, Halliday GM. A possible role for humoral immu- 455 nity in the pathogenesis of Parkinson's disease. Brain 2005;128:2665-2674. 456

[26] Graham DG. On the origin and significance of neuromelanin. Arch Pathol Lab Med 457 1979;103:359-362.

[27] Oberlander U, Pletinckx K, Dohler A, Muller N, Lutz MB, Arzberger T, et al. 459 Neuromelanin is an immune stimulator for dendritic cells in vitro. BMC Neurosci 460 2011;12:116.

[28] Koutsilieri E, Lutz MB, Scheller C. Autoimmunity, dendritic cells and relevance for 462 Parkinson's disease. J Neural Transm 2013;120:75-81. 463

[29] Wilms H, Rosenstiel P. Sievers J, Deuschl G, Zecca L, Lucius R. Activation of microglia by 464 human neuromelanin is NFkappaB dependent and involves p38 mitogen-activated 465 protein kinase: implications for Parkinson's disease. FASEB J 2003;17:500-2. 466

[30] Zhang W, Phillips K, Wielgus AR, Liu J, Albertini A, Zucca FA, et al. Neuromelanin ac- 467 tivates microglia and induces degeneration of dopaminergic neurons: implications 468 for progression of Parkinson's disease. Neurotox Res 2011;19:63-72. 469

[31] Carvey PM, McRae A, Lint TF, Ptak LR, Lo ES, Goetz CG, et al. The potential use of do- 470 pamine neuron antibody and a striatal-derived neurotropic factor as diagnostic 471 markers in Parkinson's disease. Neurology 1991;41:53-8. 472

[32] Le WD, Rowe DB, Jankovic J, Xei W, Apple SH. Effects of cerebrospinal fluid from pa- 473 tients with Parkinson disease on dopaminergic cells. Arch Neurol 1999;56:194-200. 474

[33] Chen S, Le WD, Xie WJ, Alexianu ME, Engelhardt JI, Siklos L, et al. Experimental de- 475 struction of substantia nigra initiated by Parkinson disease immunoglobulins. Arch 476 Neurol 1998;55:1075-80.

[34] He Y Le WD Appel SH. Role of FC $\gamma$ receptors in nigral cell injury induced by 478 Parkinson's disease immunoglobulin injection into mouse substantia nigra. Exp 479 Neurol 2002;176:322-7.

[35] Abramsky O, Litvin Y. Autoimmune response to dopamine-receptor as a possible 481 mechanism in the pathogenesis of Parkinson's disease and schizophrenia. Perspect 482 Biol Med 1978;22:104114.

[36] Luedtke RR, Griffin SA, Conroy SS, Jin X, Pinto A, Sesack SR. Immunoblot and immu- 484 nohistochemical comparison of murine monoclonal antibodies specific for the rat 485 D1a and D1b dopamine receptor subtypes. J Neuroimmunol 1999;101:170-87. 486

[37] Myers PR, Donlon M, McCarthy K, Livengood D, Shain W. Evidence for a dopamine 487 receptor antibody. Biochem Biophys Res Commun 1976;72:1311-8. 488

[38] Farooqui SM, Brock JW, Hamdi A, Prasad C. Antibodies against synthetic peptides 489 predicted from the nucleotide sequence of D2 receptor recognize native dopamine 490 receptor protein in rat striatum. J Neurochem 1991;57:1363-1369. 491

[39] Gibb WR, Lees AJ. The relevance of the Lewy body to the pathogenesis of idiopathic 492 Parkinson's disease. J Neurol Neurosurg Psychiatry 1988;51:745-752. 493

[40] Postuma RB, Aarsland D, Barone P, Burn DJ, Hawkes CH, Oertel W, et al. Identifying 494 prodromal Parkinson's disease: pre-motor disorders in Parkinson's disease. Mov 495 Disord 2012;27:617-626.

[41] Hely MA, Morris JGL, Reid WGJ, Trafficante R. Sydney multicenter study of 497 Parkinson's disease: non-L-dopa-responsive problems dominate at 15 years. Mov 498 Disord 2005;20:190-199.

[42] Hely MA, Reid WGJ, Adena MA, Halliday GM, Morris JGL. The Sydney multicenter 500 study of Parkinson's disease: the inevitability of dementia at 20 years. Mov Disord 501 2008;23:837-844.

[43] Lerler A, Bagic A. Olfactory pathogenesis of idiopathic Parkinson disease revisited. 503 Mov Disord 2008;23:1076-84. 504

[44] Goedert M, Spillantini MG, Del Tredici K, Braak H. 100 years of Lewy pathology. Nat 505 Rev Neurol 2012;9:13-24.

[45] Iwanaga K, Wakabayashi K, Yoshimoto M, Tomita I, Satoh H, Takashima H, et al. 507 Lewy body-type degeneration in cardiac plexus in Parkinson's and incidental Lewy 508 body diseases. Neurology 1999;52:1269-1271.

[46] Goetz CG. The history of Parkinson's disease: early clinical descriptions and neuro- 510 logical therapies. Cold Spring Harb Perspect Med 2011;1:a008862.

[47] Popat RA, Van Den Eeden SK, Tanner CM, Kamel F, Umbach DM, Marder K, et al Cof- 512 fee, ADORA2A, and CYP1A2: the caffeine connection in Parkinson's disease. Eur J 513 Neurol 2011;18:756-765.

[48] Hong Z, Shi M, Chung KA, Quinn JF, Peskind ER, Galasko D, et al. DJ-1 and $\alpha-515$ synuclein in human cerebrospinal fluid as biomarkers of Parkinson's disease. Brain 516 2010;133:713-726 
[49] Parnetti L, Castrioto A, Chiasserini D, Persichetti E, Tambasco N, El-Agnaf O, et al. Cerebrospinal fluid biomarkers in Parkinson disease. Nat Rev Neurol 2013;9:131-140.

50] Brooks DJ, Pavese N. Imaging biomarkers in Parkinson's disease. Prog Neurobiol 2011;95:614-628.

[51] Lehericy S, Sharman MA, Santos CLD, Paquin R, Gallea C. Magnetic resonance imaging of the substantia nigra in Parkinson's disease. Mov Disord 2012;27:822-830.

52] Lehericy S, Bardinet E, Poupon C, Vidailhet M, Francois C. 7 tesla magnetic resonance imaging: a closer look at substantia nigra anatomy in Parkinson's disease. Mov Disord 2014;29:1574-1581.

[53] Coelho M, Ferreira JJ. Late-stage Parkinson disease. Nat Rev Neurol 2012;8:435-442.

54] Fox SH, Katzenschlager R, Lim SY, Ravina B, Seppi K, Coelho M, et al. The Movement Disorder Society evidence-based medicine review update: treatments for the motor symptoms of Parkinson's disease. Mov Disord 2011;26:S2-41.

55] Connolly B, Lang AE. Pharmacological treatment of Parkinson's disease: a review. JAMA 2014;311:1670-1683.

[56] Ton TG, Heckbert SR, Longstreth Jr WT, Rossing MA, Kukull WA, Franklin GM, et al. Non steroidal anti-inflammatory drugs and risk of Parkinson's disease. Mov Disord 2006;21:964-969.

[57] Plane JM, Shen Y, Pleasure DE, Deng W. Prospects for minocycline neuroprotection. Arch Neurol 2010;67:1442-1448.

58] Ravina B. A randomized, double-blind, futility clinical trial of creatine and minocycline in early Parkinson disease. Neurology 2006;66:664-671.

[59] Thevenet J, Pescini Gobert R, van Huijsduijnen R H, Wiessner C, YJ S. Regulation of LRRK2 expression points to a functional role in human monocyte maturation. PLoS One 2011;6:e21519.
[60] Moehle MS, Webber PJ, Tse T, Sukar N, Standaert DG, DeSilva TM, et al. LRRK2 inhibi- 543 tion attenuates microglial inflammatory responses. J Neurosci 2012;32:1602-1611. 544

[61] Wenz T. PGC- $1 \alpha$ activation as a therapeutic approach in mitochondrial disease. 545 IUBMB Life 2009;61:1051-1062.

[62] Hondares E, Pineda-Torra I, Iglesias R, Staels B, Villarroya F, Giralt M. PPAR $\delta$, but not 547 PPAR $\alpha$, activates PGC- $1 \alpha$ gene transcription in muscle. Biochem Biophys Res 548 Commun 2007;354:1021-1027.

[63] Pardo R, Enguix N, Lasheras J, Feliu JE Kralli A, Villena JA Rosiglitazone-induced mi- 550 tochondrial biogenesis in white adipose tissue is independent of peroxisome 551 proliferator- activated receptor $\gamma$ coactivator- $1 \alpha$. PLoS One 2011;6:e26989. 552

[64] Suwa M, Egashira T, Nakano H, Sasaki H, Kumagai S. Metformin increases the PGC- 553 $1 \alpha$ protein and oxidative enzyme activities possibly via AMPK phosphorylation in 554 skeletal muscle in vivo. J Appl Physiol 2006;101:1685-92.

[65] Chowanadisai W, Bauerly KA, Tchaparian E, Wong A, Cortopassi GA, Rucker RB. 556 Pyrroloquinoline quinine stimulates mitochondrial biogenesis through cAMP re- 557 sponse element-binding protein phosphorylation and increased PGC- $1 \alpha$ expression. 558 J Biol Chem 2010;285:142-52.

[66] Irrcher I, Ljubicic V, Kirwan AF, Hood DA. AMP activated protein kinase-regulated acti- 560 vation of the PGC-1alpha promoter in skeletal muscle cells. PLoS One 2008;3:e3614. 561

[67] Lagouge M, Argmann C, Gerhart-Hines Z, Meziane H, Lerin C, Daussin F, et al. Resver- 562 atrol improves mitochondrial function and protects against metabolic disease by ac- 563 tivating SIRT1 and PGC-1alpha. Cell 2006;127:1109-1122.

[68] Shoenfeld Y, Blank M, Abu-Shakra M, Amital H, Barzilai O, Berkun Y, et al. The mosaic 565 of autoimmunity: prediction, autoantibodies and therapy in autoimmune disease. 566 IMAJ 2008;10:13-9. 